\title{
Beam propagation through atmospheric turbulence using an altitude-dependent structure profile with non-uniformly distributed phase screens
}

\author{
Yousef K. Chahine, Sarah A. Tedder, Brian E. Vyhnalek, and Adam C. Wroblewski \\ NASA Glenn Research Center, Cleveland, OH, USA
}

\begin{abstract}
Modeling the effects of atmospheric turbulence on optical beam propagation is a key element in the design and analysis of free-space optical communication systems. Numerical wave optics simulations provide a particularly useful technique for understanding the degradation of the optical field in the receiver plane when the analytical theory is insufficient for characterizing the atmospheric channel. Motivated by such an application, we use a splitstep method modeling the turbulence along the propagation path as a series of thin random phase screens with modified von Kármán refractive index statistics using the Hufnagel-Valley turbulence profile to determine the effective structure constant for each screen. In this work, we employ a space-to-ground case study to examine the irradiance and phase statistics for both uniformly and non-uniformly spaced screens along the propagation path and compare to analytical results. We find that better agreement with the analytical theory is obtained using a non-uniform spacing with the effective structure constant for each screen chosen to minimize its contribution to the scintillation in the receiver plane. We evaluate this method as a flexible alternative to other standard layered models used in astronomical imaging applications.
\end{abstract}

Keywords: Wave optics, atmospheric turbulence, phase screen, Hufnagel-Valley

\section{INTRODUCTION}

Modeling laser beam propagation through a turbulent atmospheric channel provides critical insight into the expected wavefront behavior at the aperture of an optical receiver. This behavior should be understood early in the optical system's development process in order to adequately predict communication link budgets and define optical system design requirements. Accurate beam propagation modeling through turbulent media, specifically Earth's atmosphere, is required for predicting performance in a variety of optical link scenarios such as spacecraft-to-ground, spacecraft-to-aircraft, aircraft-to-aircraft, aircraft-to-ground, and ground-to-ground.

Analytical methods are often used for producing high-level link budgets for atmospheric channels when the communications equipment is already well-characterized; however, Monte Carlo numerical wave optics simuluations provide a useful tool facilitating the analysis of uncharacterized optical receiver and transmitter designs that are expected to operate under a variety of atmospheric conditions. The basic method for such simulations is reviewed in Section 2 and is well-documented in the literature. The split-step method discretizes the propagation path as an alternating sequence of vacuum propagations and thin phase screens representing the cumulative effect of the turbulence on the optical wave propagating through a corresponding extended volume of atmosphere.

For slant path atmospheric channels it is important to correctly model the variations in the strength of the optical turbulence along the propagation path which is known to vary appreciably with altitude. A number of methods for the discretization of such path-dependent turbulence profiles are discussed in Section 3; however, a thorough study of this problem does not appear in the literature. While a completely general analysis is beyond the scope of this paper, we give in this report an analysis of several generally applicable discretization schemes evaluated by comparing the irradiance and phase statistics of the numerically propagated optical fields to those expected from the analytical theory based on propagation through a continuously varying turbulence profile. In particular, we are interested in identifying a general method which is readily adaptable to handle the variety of slant path turbulence profiles associated to the optical link scenarios mentioned above and which requires minimal case-by-case supervision. 


\section{BACKGROUND}

\subsection{Split-step beam propagation method}

The split-step beam propagation method is a numerical method for solving the linear Schrödinger equation

$$
i \frac{\partial u}{\partial z}=-\frac{1}{2 k}\left(\frac{\partial^{2}}{\partial x^{2}}+\frac{\partial^{2}}{\partial y^{2}}\right) u-k n_{1}(x, y, z) u
$$

for a given initial condition $u(x, y, 0)=f(x, y)$, where $k=2 \pi / \lambda$ is a real constant and $n_{1}(x, y, z) \ll 1$ is a real-valued function typically representing small fluctuations in the refractive index of a background medium. Following Spivack and Uscinski, ${ }^{1}$ the method can be described as an operator-splitting method obtained by rewriting the above equation in the form

$$
\frac{\partial u}{\partial z}=i(A+B) u
$$

where we have split the Hermitian operator $H=A+B$ into a free-space operator $A=(1 / 2 k)\left[\partial^{2} / \partial x^{2}+\partial^{2} / \partial y^{2}\right]$ and a scattering operator $B=k n_{1}$, respectively. Formally, we may write the solution $u(x, y, L)$ in the plane $z=L$ as $u(x, y, L)=U(L, 0) u(\cdot, 0)$ where the evolution operator $U\left(z_{1}, z_{0}\right)$ is the unitary operator defined by the 'time'-ordered exponential

$$
U\left(z_{1}, z_{0}\right)=\lim _{N \rightarrow \infty} \prod_{m=1}^{N} e^{i H\left(z_{0}+m \Delta z / N\right) \cdot \Delta z / N}
$$

with $\Delta z=z_{1}-z_{0}$. The split-step algorithm to propagate the field $u(x, y, 0)$ can then be understood as a method to approximate this evolution operator by first choosing a segmentation of the propagation path $z_{0}=0<z_{1}<$ $\ldots<z_{n}=L$ and writing

$$
u(\cdot, L)=U\left(z_{n}, z_{n-1}\right) U\left(z_{n-1}, z_{n-2}\right) \ldots U\left(z_{1}, z_{0}\right) u(\cdot, 0)
$$

and then applying two successive approximations (known together as the "phase screen approximation")

$$
\begin{aligned}
U\left(z_{j+1}, z_{j}\right) & \simeq \exp \left(i \int_{z_{i}}^{z_{i+1}} H(z) d z\right) \\
\exp \left(i \int_{z_{j}}^{z_{j+1}} H(z) d z\right) & \simeq \exp \left(i \alpha_{j} \Delta z_{j} A\right) \cdot \exp \left(i \int_{z_{j}}^{z_{j+1}} B(z) d z\right) \cdot \exp \left(i\left(1-\alpha_{j}\right) \Delta z_{j} A\right)
\end{aligned}
$$

where $\Delta z_{j}=z_{j+1}-z_{j}$ and $0 \leq \alpha_{j} \leq 1$ is a freely chosen parameter associated to each interval $\left[z_{j}, z_{j+1}\right]$. In this paper we are interested in understanding the dependence of the error introduced by these approximations on the choice of segmentation parameters $z_{j}, \alpha_{j}$ given certain assumptions on the refractive index fluctuations $n_{1}$ outlined in Section 2.3.

In the context of scalar diffraction theory it can be shown that a monochromatic traveling optical wave $u e^{i k z}$ with narrow angular spread along the propagation axis ( $z$-axis) satisfies equation (1) provided the refractive index $n=1+n_{1}$ has sufficiently small fluctuations $n_{1} \ll 1 .^{2}$ In this context the split-step method can be interpreted as a layered model for the medium as illustrated in Figure 1, where the effect of the refractive index fluctuations in each layer $\left[z_{j}, z_{j+1}\right]$ is represented by a single phase screen $e^{i \phi(x, y)}$ where $\phi$ is given in (4) by

$$
\phi(x, y)=\int_{z_{j}}^{z_{j+1}} B(z) d z=k \int_{z_{j}}^{z_{j+1}} n_{1}(x, y, z) d z
$$

and describes the variations of the optical path length along rays parallel to the propagation axis. On the other hand, the solution to $u(\cdot, \Delta z)=\exp (i \Delta z A) u(\cdot, 0)$ is given by the Fresnel integral

$$
u(\boldsymbol{\rho}, \Delta z)=2 i k \iint G_{p}\left(\boldsymbol{\rho}, \Delta z ; \boldsymbol{\rho}^{\prime}, 0\right) u\left(\boldsymbol{\rho}^{\prime}, 0\right) d^{2} \boldsymbol{\rho}^{\prime}
$$

where $\boldsymbol{\rho}=(x, y)$ denotes the transverse coordinates and $G_{p}\left(\boldsymbol{\rho}, z ; \boldsymbol{\rho}^{\prime}, z^{\prime}\right)=-e^{i k\left(\boldsymbol{\rho}-\boldsymbol{\rho}^{\prime}\right)^{2} / 2\left(z-z^{\prime}\right)} / 4 \pi\left(z-z^{\prime}\right)$ is the parabolic equation Green function. This integral can be found numerically very efficiently using a fast Fourier transform and thus the split-step method described above yields an efficient numerical method for solving equation (1) by the alternating application of the free-space evolution operator and the phase screens as specified in (2),(3), and (4). 


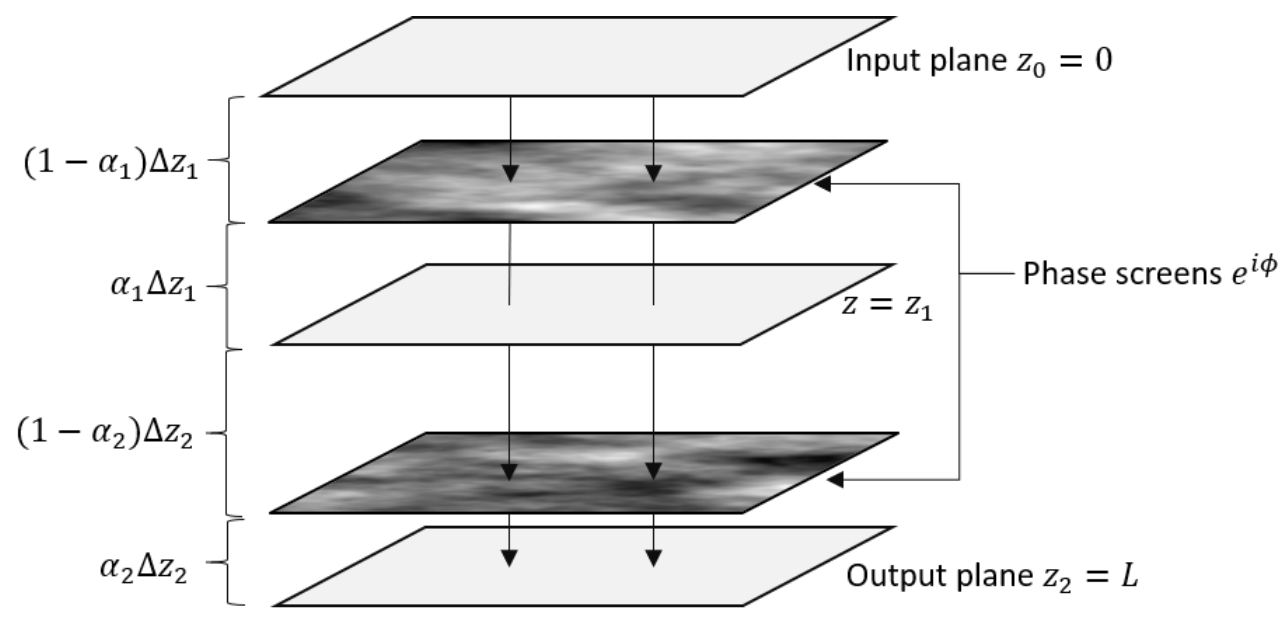

Figure 1. Schematic of the split-step beam propagation method.

\subsection{Phase screen statistics}

The split-step method presented above is particularly useful for Monte Carlo wave optics simulations where one is interested in obtaining solutions for a large number of randomly generated fields $n_{1}$ with specified spatial covariance. If $n_{1}$ represents fluctuations of the refractive index in the atmosphere due to turbulence, it is convenient to represent its covariance in terms of a power spectral density $\Phi_{n}(\boldsymbol{\kappa}, z)$ via the Fourier transform relation

$$
\left\langle n_{1}\left(\mathbf{r}_{1}\right) n_{1}\left(\mathbf{r}_{2}\right)\right\rangle=\iiint_{-\infty}^{\infty} d^{3} \boldsymbol{\kappa} \cdot e^{i \boldsymbol{\kappa} \cdot\left(\mathbf{r}_{2}-\mathbf{r}_{1}\right)} \Phi_{n}\left(\boldsymbol{\kappa}, \frac{z_{1}+z_{2}}{2}\right)
$$

where $\mathbf{r}_{i}=\left(x_{i}, y_{i}, z_{i}\right)$ and we further assume $n_{1}\left(\mathbf{r}_{i}\right)$ has Gaussian statistics with zero mean and variance $\sigma_{n}^{2}\left(\mathbf{r}_{i}\right)$.

One of the benefits of using the split-step method is that one need not generate the full three-dimensional refractive index field $n_{1}(x, y, z)$; it suffices to generate the related 2-dimensional phase screens $\phi(x, y)$ defined by equation (5). In order to obtain the power spectral density $\Phi_{\phi}$ for the phase, one uses (5) to write

$$
\left\langle\phi\left(\boldsymbol{\rho}_{1}\right) \phi\left(\boldsymbol{\rho}_{2}\right)\right\rangle=k^{2} \int_{z_{i}}^{z_{i+1}} \int_{z_{i}}^{z_{i+1}}\left\langle n_{1}\left(\boldsymbol{\rho}_{1}, \zeta_{1}\right) n_{1}\left(\boldsymbol{\rho}_{2}, \zeta_{2}\right)\right\rangle d \zeta_{1} d \zeta_{2}
$$

where $\boldsymbol{\rho}_{i}=\left(x_{i}, y_{i}\right)$ denotes the transverse coordinates. Substituting (7) for the integrand yields

$$
\left\langle\phi\left(\boldsymbol{\rho}_{1}\right) \phi\left(\boldsymbol{\rho}_{2}\right)\right\rangle=\iint_{-\infty}^{\infty} d^{2} \boldsymbol{\kappa}_{\rho} \cdot e^{i \boldsymbol{\kappa}_{\rho} \cdot\left(\boldsymbol{\rho}_{2}-\boldsymbol{\rho}_{1}\right)} \Phi_{\phi}\left(\boldsymbol{\kappa}_{\rho}\right),
$$

where

$$
\Phi_{\phi}\left(\boldsymbol{\kappa}_{\rho}\right)=k^{2} \int_{z_{i}}^{z_{i+1}} \int_{z_{i}}^{z_{i+1}} \int_{-\infty}^{\infty} e^{i \kappa_{z}\left(\zeta_{2}-\zeta_{1}\right)} \Phi_{n}\left(\boldsymbol{\kappa}_{\rho}, \kappa_{z}, \frac{\zeta_{1}+\zeta_{2}}{2}\right) d \kappa_{z} d \zeta_{1} d \zeta_{2} .
$$

In Appendix A.1 it is shown that if the path-dependence is of the form $\Phi_{n}(\boldsymbol{\kappa}, z)=\sigma_{n}^{2}(z) \hat{\Phi}_{n}(\boldsymbol{\kappa})$ then the integral above can be evaluated to obtain

$$
\Phi_{\phi}\left(\boldsymbol{\kappa}_{\rho}\right) \simeq 2 \pi k^{2} \hat{\Phi}_{n}\left(\boldsymbol{\kappa}_{\rho}, \kappa_{z}=0\right) \int_{z_{i}}^{z_{i+1}} \sigma_{n}^{2}(z) d z
$$

provided that $\Delta z \gg L_{0}$ where $L_{0}$ is the correlation length of the refractive index fluctuations $n_{1}$ and provided that the fluctuations are not primarily localized within a distance $L_{0}$ of the segment boundary $z=z_{i}$ and $z=z_{i+1}$. Note that we need not invoke the Markov approximation here as is traditional in deducing (10), though the Markov limit is still present in the assumption $\Delta z \gg L_{0}$ and is relevant in treating (or ignoring) correlations between neighboring phase screens. 
Henceforth, we shall assume the path-dependence of the power spectral density $\Phi_{n}$ is of the form above where $\sigma_{n}^{2}(z)$ is the variance of the random variable $n_{1}(x, y, z)$ and $\hat{\Phi}_{n}$ is the power spectral density normalized by the variance $\sigma_{n}^{2}(z)$. Furthermore, in order to emphasize the dependence of the phase $\phi$ on the integration segment we introduce the normalized phase $\hat{\phi}=\phi / k \Sigma_{n}$ where

$$
\Sigma_{n}^{2}=\int_{z_{i}}^{z_{i+1}} \sigma_{n}^{2}(z) d z
$$

is the integrated variance along the propagation segment $\left[z_{i}, z_{i+1}\right]$, noting that the normalized phase $\hat{\phi}$ has power spectral density $\hat{\Phi}_{\phi}\left(\boldsymbol{\kappa}_{\rho}\right)=2 \pi \hat{\Phi}_{n}\left(\boldsymbol{\kappa}_{\rho}, 0\right)$.

\subsection{Refractive index model}

In this work, we assume the power spectral density of refractive index fluctuations is that of a turbulent medium under the assumption of locally homogeneous and isotropic turbulence

$$
\Phi_{n}(\kappa, z)=0.033 C_{n}^{2}(z) \kappa^{-11 / 3}, \quad \frac{1}{L_{0}}<\kappa<\frac{1}{l_{0}}
$$

where $l_{0}$ and $L_{0}$ are the inner and outer scales of the turbulent eddies, respectively. In this context, the variance $\sigma_{n}^{2}(z)$ of the refractive index fluctuations is customarily given in terms of the refractive index structure constant $C_{n}^{2}(z)$, which is proportional to the variance with a constant of proportionality which depends on the inner and outer scales $l_{0}$ and $L_{0}$ in a manner determined by (7). A simple model for the inner and outer scales is included by using in place of (11) the modified von Kármán power spectral density (PSD)

$$
\Phi_{n}(\kappa, z)=\frac{0.033 C_{n}^{2}(z) e^{-\kappa^{2} / \kappa_{m}^{2}}}{\left(\kappa^{2}+\kappa_{0}^{2}\right)^{11 / 6}}
$$

where $\kappa_{m}=5.92 / l_{0}$ and $\kappa_{0}=2 \pi / L_{0}$, which gives a very rough approximation to empirical data outside of the inertial sub-range $1 / L_{0}<\kappa<1 / l_{0}$ and removes the divergence in expression (11) as $\kappa \rightarrow 0$.

It is well-known that turbulence in the Earth's atmosphere depends on altitude and other atmospheric conditions in a complex manner with the most severe turbulence located within the first few kilometers above ground level. To account for this, we assume the empirically-based Hufnagel-Valley vertical turbulence profile giving the refractive index structure constant as a function of the height $h$ in meters above ground level via

$$
C_{n}^{2}(h)=0.00594\left(\frac{v}{27}\right)^{2}(h / 10000)^{10} e^{-h / 1000}+2.7 \cdot 10^{-16} e^{-h / 1500}+C_{0} e^{-h / 100}
$$

where the tunable parameters $v$ and $C_{0}$ represent the root mean square (rms) upper atmospheric wind speed and the value of $C_{n}^{2}$ at ground level, respectively. ${ }^{3}$

\subsection{Rytov approximation}

Our aim is to evaluate numerically the performance of different splitting parameters $z_{i}, \alpha_{i}$ in the split-step method for solving the parabolic wave equation (1) assuming the refractive index fluctuation $n_{1}(x, y, z)$ represents a stochastic field with zero mean, power spectral density (12), and path-dependent variance $\sigma_{n}^{2}(z)=\left\langle n_{1}(x, y, z)^{2}\right\rangle$ determined by (13). The primary tool used in this evaluation will be perturbative solutions to (1) furnished under these assumptions by the Rytov method.

The Rytov method is a perturbation-theoretic technique which allows one to approximate statistical moments of the solutions $u(\boldsymbol{\rho}, L)$ of the stochastic parabolic wave equation (1). The substitution $u=e^{\psi}$ transforms (1) into a Riccati-type equation

$$
2 i k \frac{\partial \psi}{\partial z}+(\nabla \psi)^{2}+\nabla_{\rho}^{2} \psi+2 k^{2} n_{1}=0
$$


which trades the coupled stochastic term $n_{1} u$ for a nonlinear term (here, $\nabla_{\rho}$ denotes the gradient in the transverse coordinates). Expanding $\psi(\boldsymbol{\rho}, L)$ in a perturbation series $\psi=\psi_{0}+\psi_{1}+\psi_{2}+\ldots$ where $u_{0}=e^{\psi_{0}}$ is the unperturbed free-space solution of (1) given by the Fresnel integral (6) and

$$
\begin{aligned}
& \psi_{1}(\boldsymbol{\rho}, L)=-k^{2} \int_{0}^{L} \iint_{-\infty}^{\infty} G_{p}\left(\boldsymbol{\rho}, L, \boldsymbol{\rho}^{\prime}, z\right) n_{1}\left(z, \boldsymbol{\rho}^{\prime}\right)\left(\frac{u_{0}\left(\boldsymbol{\rho}^{\prime}, z\right)}{u_{0}(\boldsymbol{\rho}, L)}\right) d^{2} \boldsymbol{\rho}^{\prime} d z \\
& \psi_{2}(\boldsymbol{\rho}, L)=-\int_{0}^{L} \iint_{-\infty}^{\infty} G_{p}\left(\boldsymbol{\rho}, L, \boldsymbol{\rho}^{\prime}, z\right) n_{1}\left(z, \boldsymbol{\rho}^{\prime}\right)\left[\nabla_{\rho} \psi_{1}\left(\boldsymbol{\rho}^{\prime}, z\right)\right]^{2}\left(\frac{u_{0}\left(\boldsymbol{\rho}^{\prime}, z\right)}{u_{0}(\boldsymbol{\rho}, L)}\right) d^{2} \boldsymbol{\rho}^{\prime} d z
\end{aligned}
$$

yield the first and second order Rytov approximations. ${ }^{4}$

Denoting the log-amplitude and phase perturbations by $\chi+i S=\psi_{1}+\psi_{2}+\ldots$ one can obtain approximations for various statistical moments such as the log-amplitude variance $\left\langle\chi^{2}\right\rangle$, mean irradiance $\langle I(\boldsymbol{\rho}, L)\rangle=$ $\left|u_{0}(\boldsymbol{\rho}, L)\right|^{2}\left\langle e^{2 \chi(\boldsymbol{\rho}, L)}\right\rangle$, etc. Such approximations shall be introduced as needed in the following, and are known to be valid provided the irradiance fluctuations are sufficiently small $\left(\sigma_{I}^{2}=\exp \left(4\left\langle\chi^{2}\right\rangle\right)-1 \lesssim 0.25\right){ }^{2}$

\section{DISCRETIZATION OF THE PROPAGATION PATH}

For very small sampling grids, one can minimize the discretization error in the approximations (3) and (4) by simply increasing the number of phase screens. For example, using up to 128 phase screens, Coles and Frehlich found that as the screen spacing becomes small the error in the normalized intensity is linearly proportional to the segment length $\Delta z$ represented by each screen, in agreement with the original analysis of Spivack and Uscinski. ${ }^{5}$ However, for larger $N \times N$ sampling grids (e.g. $N \geq 2048$ ) each additional screen adds significant computational time to the beam propagation algorithm and it is thus desirable to optimize the segmentation to minimize both the error and the number of phase screens.

It is well-known that for an extended region to be accurately represented by a single phase screen the total scattering strength of the screen must be sufficiently small. ${ }^{6}$ Extending the analysis of Spivack and Uscinski, we provide in the appendix a partial analysis of the approximation (4) including both the segment length $\Delta z$ and the scattering strength which is shown by (10) to be dependent on the integrated turbulence strength $\Sigma_{n}$. Loosely speaking, the phase screen approximation becomes insufficient when the propagation distance after appreciable wavefront distortions develop is too large to ignore the redistribution of energy due to propagation of the perturbed wavefront. However, a preliminary mathematical analysis in Appendix A.2 is as yet insufficient to determine precisely the subtle dependence of the phase screen approximation on the combination of the scattering strength $\Sigma_{n}$ and propagation distance $\Delta z$.

This complex behavior has prompted several suggestions of methods for segmenting the propagation path to improve the phase screen approximation. For example, Martin and Flattè suggest that the contribution of each segment to the total scintillation index (as measured by the Rytov variance) should not be too large. ${ }^{6}$ Another common approach is to attempt to choose both the segmentation $z_{i}$ and the weights $\alpha_{i}$ in (4) in such a way that the discrete moments match the continuous moments

$$
\sum_{i=1}^{n} C_{n_{i}}^{2} \zeta_{i}^{m}=\int_{0}^{L} z^{m} C_{n}^{2}(z) d z
$$

where $C_{n_{i}}^{2}=\int_{z_{i}}^{z_{i+1}} C_{n}^{2}(z) d z$ is the integrated turbulence and $\zeta_{i}=\left(1-\alpha_{i}\right) z_{i}+\alpha_{i} z_{i+1}$ is the location of the $i$-th phase screen. ${ }^{7}$ With $n$ screens this latter approach matches the first $n-1$ moments, but leaves the placement of the screens as a freely specifiable parameter; however, for many choices of screen placement the solution of the moment equations above leads to unphysical negative weights $C_{n_{i}}^{2}$. For example, when this method was introduced by Troxel et al. for simplifying the mathematical analysis of imaging through turbulence, ${ }^{8}$ it was determined on an ad hoc basis that a four-layer model was sufficient with screens placed at $200 \mathrm{~m}, 2 \mathrm{~km}, 10 \mathrm{~km}$, and $18 \mathrm{~km}$, reportedly matching the optical transfer function for propagation through the atmosphere to within $1 \%$ of the continuous $C_{n}^{2}$ profile.

In attempting to model a variety of propagation channels, particularly those which may evolve dynamically (e.g. in the case of links to and from an aircraft), it is desirable to have a general and flexible algorithm for 
obtaining an optimal discretization of the slant path turbulence profile which requires minimal case-by-case supervision. As a step in this direction, we make in this paper a comparative study of various general algorithms for determining the segmentation of the propagation path $\Delta z_{i}, \alpha_{i}$. In particular, we compare algorithms which segment the path based on (1) minimizing the variance of the phase for each screen (i.e. minimizing $\Sigma_{n}$ ), (2) minimizing the propagation distance $\Delta z$ represented by each screen (uniform segments), and (3) minimizing the contribution of each screen to the total scintillation.

For the latter, we note that the on-axis log-amplitude variance for a Gaussian beam wave in weak turbulence with modified von Kármán refractive index statistics can be found via the Rytov method to be given by ${ }^{9}$

$$
\left\langle\chi(0)^{2}\right\rangle=-0.033 \pi^{2} k^{2} \Gamma(-5 / 6) \int_{0}^{L} C_{n}^{2}(z) \cdot G(z) d z
$$

where $G(z)$ is a spatial filter function which depends on the size and location of the beam waist and gives a larger weight to turbulence near the source. Specifically, this function is given by $G(z)=\operatorname{Re}\left[f(z)^{5 / 6}\right]-\operatorname{Re}[f(z)]^{5 / 6}$ where

$$
f(z)=i \frac{z-q_{0}}{L-q_{0}}\left(\frac{L-z}{k}\right)+\frac{1}{\kappa_{m}^{2}}
$$

and $q_{0}$ is the complex beam parameter specifying the Gaussian beam in the source plane according to $u(\rho, 0)=$ $E_{0} \exp \left(i k \rho^{2} / 2 q_{0}\right)$. This reduces to the Rytov variance as the Gaussian beam approaches a plane wave. For segmentation method (3), we thus choose our segments $\left[z_{i}, z_{i+1}\right]$ so that each segment contributes equally (hence, minimally) to the integral (14).

For methods (1)-(3) considerations in Appendix A.2 suggest that one can minimize the error introduced in (4) by placing the phase screens at the midpoint of each segment analogous to Strang splitting with $\alpha_{i}=1 / 2$; however, this neglects the original error introduced by the phase screen approximation in (3). Another reasonable option is to locate the screen in each segment at the turbulent center of mass defined by

$$
\zeta_{i}=\frac{\int_{z_{i}}^{z_{i+1}} z C_{n}^{2}(z) d z}{\int_{z_{i}}^{z_{i+1}} C_{n}^{2}(z) d z} .
$$

To compare the two options for locating the screen within each segment we examine segmentation method (3) with both centered, and center-of-mass (COM) located screens. In the following, we shall refer to the discretization algorithms (1),(2), and (3) as "phase-minimized" (PM), "distance-minimized" (DM), and "scintillationminimized" (SM), respectively, with the latter further specified as centered or center-of-mass depending on the location of the phase screens. The four-layer model of Troxel et al. described above shall be referred to as the "moment-matched" (MM) discretization.

\section{RESULTS OF NUMERICAL SIMULATION}

To examine the general methods described in Section 3, we consider as a case study a $24 \mathrm{~km}$ vertical propagation path representing a space-to-ground atmospheric channel (we remark that the $24 \mathrm{~km}$ altitude is not chosen to represent the altitude of a transmitter/receiver but rather represents the onset of the refractive index fluctuations for a beam propagating from space - such fluctuations are assumed to vanish above $24 \mathrm{~km}$ when applying the Hufnagel-Valley model ${ }^{10}$ ). The path is segmented using 4 phase screens so that we may compare the three general algorithms discussed above to the moment-matched four-layer model introduced by Troxel et al. A summary of the different discretization algorithms applied to this propagation path is given in Table 1 and Figures 2-3. In this study we consider several turbulence levels obtained from the Hufnagel-Valley model by adjusting the ground level refractive index structure constant $C_{0}$ in (13); the rms upper atmospheric wind speed is fixed at $v=21 \mathrm{~m} / \mathrm{s}$ as in the Hufnagel-Valley $5 / 7$ model commonly used to represent optical turbulence during daytime. ${ }^{3}$

To compute random draws of the phase screens with the desired spatial statistics (10), we use the subharmonic phase screen method shown by Frehlich ${ }^{11}$ to yield higher fidelity statistics at large spatial scales compared to a Fourier transform method which only selects random draws for Fourier coefficients within the frequency range imposed by the spatial grid. For these simulations we have used subharmonic phase screens of order $p=3 .{ }^{11} \mathrm{We}$ 

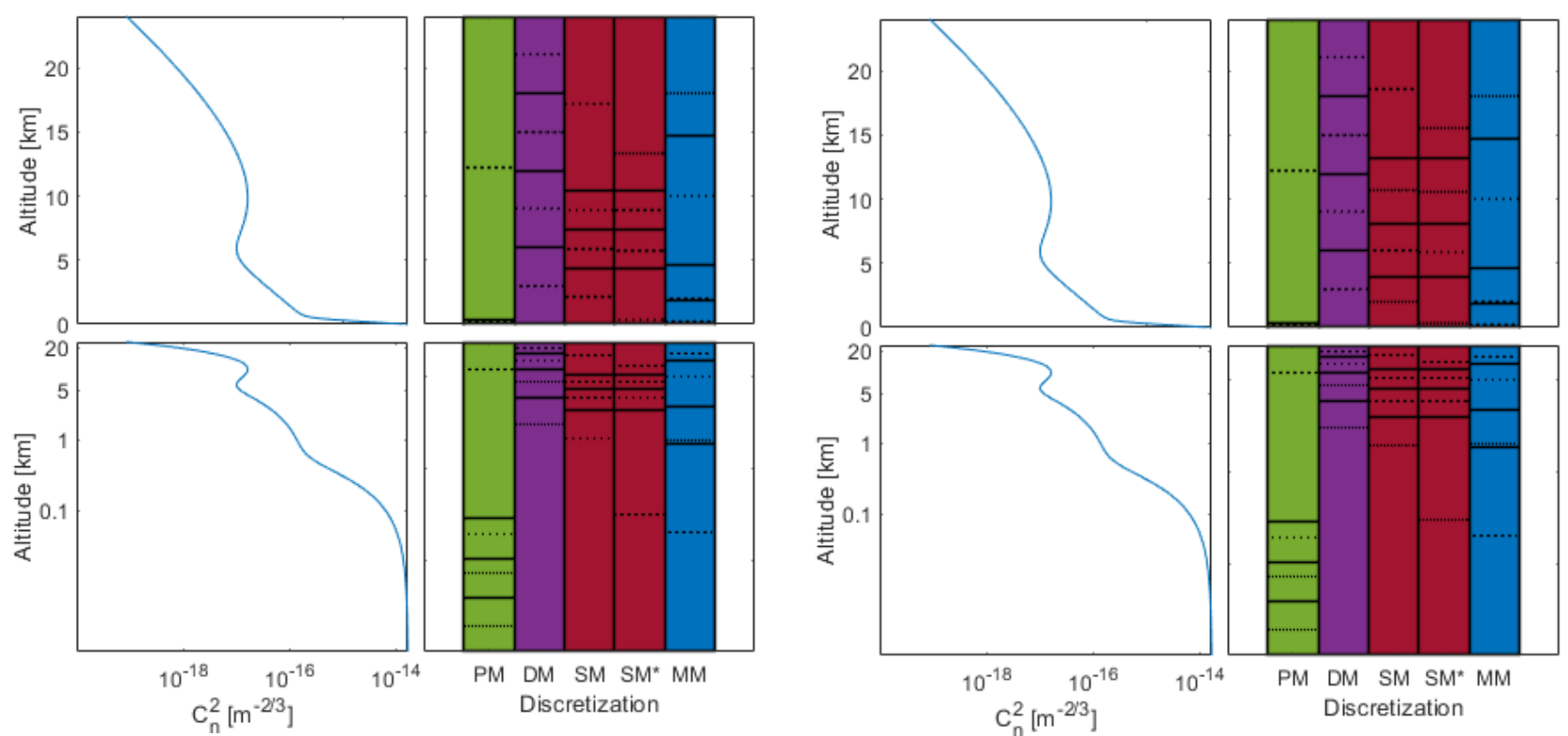

Figure 2. Discretization of the HV-21 downlink propagation path with $C_{0}=1.7 \cdot 10^{-14} \mathrm{~m}^{-2 / 3}$. Solid lines represent segment boundaries and dotted lines the locations of the
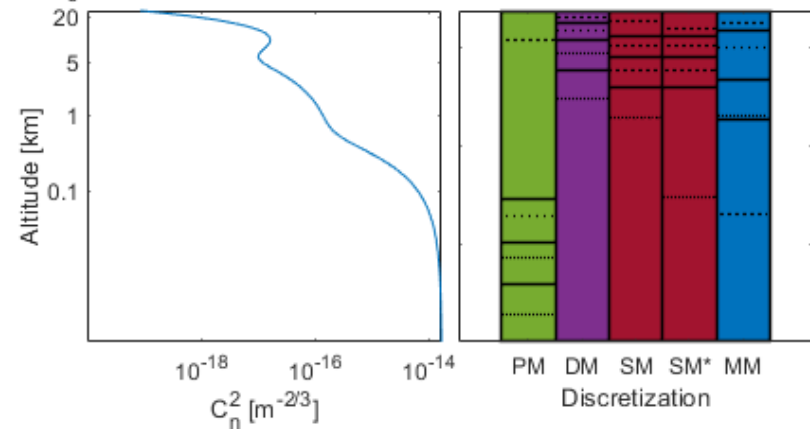

Figure 3. Discretization of the HV-21 uplink propagation path with $C_{0}=1.7 \cdot 10^{-14} \mathrm{~m}^{-2 / 3}$. phase screens. Bottom: Logarithmic altitude scale.

\begin{tabular}{|c|c|c|c|c|c|c|c|c|c|c|c|c|c|c|}
\hline \multirow[b]{3}{*}{ Type } & \multicolumn{7}{|c|}{$\mathrm{HV}-21, C_{0}=1.7 \cdot 10^{-14} \mathrm{~m}^{-2 / 3}$} & \multicolumn{7}{|c|}{$\mathrm{HV}-21, C_{0}=6.8 \cdot 10^{-14} \mathrm{~m}^{-2 / 3}$} \\
\hline & \multicolumn{3}{|c|}{ Segment Boundaries } & \multicolumn{4}{|c|}{ Screen Locations } & \multicolumn{3}{|c|}{ Segment Boundaries } & \multicolumn{4}{|c|}{ Screen Locations } \\
\hline & $z_{1}$ & $z_{2}$ & $z_{3}$ & $\alpha_{1}$ & $\alpha_{2}$ & $\alpha_{3}$ & $\alpha_{4}$ & $z_{1}$ & $z_{2}$ & $z_{3}$ & $\alpha_{1}$ & $\alpha_{2}$ & $\alpha_{3}$ & $\alpha_{4}$ \\
\hline$\overline{\mathrm{PM}}$ & $39 n$ & $103 \mathrm{~m}$ & 2891 & 0.5 & 0.5 & & & & $77 \mathrm{n}$ & 162 & 0.5 & 0.5 & 0.5 & 0.5 \\
\hline & $\mathrm{km}$ & $12 \mathrm{~km}$ & $18 \mathrm{l}$ & 05 & 0 . & 0 & & $\mathrm{kn}$ & $12 \mathrm{~km}$ & 18 & 0.5 & 0 & & 0.5 \\
\hline & $\mathrm{rm}$ & $8.07 \mathrm{~km}$ & 13.2 & 0.5 & 0. & 0 . & & $\mathrm{km}$ & $7.82 \mathrm{~km}$ & 13 & 0.5 & 0 & & 0.5 \\
\hline & & 8. & & 0 & 0 . & & & & & & 6 & 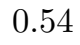 & & \\
\hline & $2 \mathrm{~km}$ & $4.69 \mathrm{~km}$ & $14.8 \mathrm{~km}$ & 0.89 & 0.9 & & & $750 \mathrm{~m}$ & $5.28 \mathrm{~km}$ & 14 & 0.87 & 0.7 & 0. & 0.6 \\
\hline & $\overline{\mathrm{j} m}$ & $\overline{1} \overline{6 . \overline{7}} \overline{\mathrm{km}}$ & $\overline{1} \overline{9} . \overline{7} \mathrm{~km}$ & $\overline{0} . \overline{5}$ & $\overline{0}$ & & & $\overline{13.7} \mathrm{~km}$ & $\overline{16.7} \mathrm{~km}$ & & $0 . \overline{5}$ & & & 0.5 \\
\hline $\mathrm{SM}^{*}-\mathrm{DL}$ & $13.6 \mathrm{~km}$ & $16.7 \mathrm{~km}$ & $19.7 \mathrm{~km}$ & 0.22 & 0.52 & 0.48 & 0.07 & $13.7 \mathrm{~km}$ & $16.7 \mathrm{~km}$ & $19.8 \mathrm{~km}$ & 0.22 & 0.52 & 0.48 & 0.0 \\
\hline
\end{tabular}

Table 1. Segmentation parameters produced by the phase-minimized (PM), distance-minimized (DM), scintillationminimized (SM), center of mass scintillation-minimized ( $\left.\mathrm{SM}^{*}\right)$, and moment-matched (MM) discretization algorithms for a $24 \mathrm{~km}$ vertical uplink propagation path using the Hufnagel-Valley profile. The scintillation-minimizing method is the only asymmetric method and also depends on the input beam; the uplink and downlink parameters are given for a $6 \mathrm{~cm}$ and $2 \mathrm{~m}$ collimated beam, respectively.

take for the inner and outer scales of the modified von Kármán spectrum the values $l_{0}=4 \mathrm{~mm}$ and $L_{0}=100 \mathrm{~m}$ representing typical values for turbulence in the Earth's atmosphere.

The performance of the different discretization schemes is evaluated by examining the statistics of the propagated optical fields $u(\boldsymbol{\rho})$ in the receiver plane and comparing to those expected from the Rytov theory with continuous $C_{n}^{2}$ profile. One measure we consider is the averaged wave structure function $D(r)$ given by averaging the wave structure function $D(\boldsymbol{\rho},-\boldsymbol{\rho})$ over all pairs of points $(\boldsymbol{\rho},-\boldsymbol{\rho})$ located symmetrically about the beam axis at a separation distance $r=|\boldsymbol{\rho}-(-\boldsymbol{\rho})|=2|\boldsymbol{\rho}|$. Recall that the wave structure function is the sum of the phase and log-amplitude structure functions $D_{\chi}$ and $D_{S}$ defined by

$$
D_{\chi}\left(\mathbf{r}_{1}, \mathbf{r}_{2}\right)=\left\langle\left[\chi\left(\mathbf{r}_{1}\right)-\chi\left(\mathbf{r}_{2}\right)\right]^{2}\right\rangle, \quad D_{S}\left(\mathbf{r}_{1}, \mathbf{r}_{2}\right)=\left\langle\left[S\left(\mathbf{r}_{1}\right)-S\left(\mathbf{r}_{2}\right)\right]^{2}\right\rangle, .
$$

For locally homogeneous and isotropic turbulence the wave structure function $D(\boldsymbol{\rho},-\boldsymbol{\rho})$ for a Gaussian beam wave at axisymmetrically located points depends only on the separation distance $r=2|\boldsymbol{\rho}|$ and is given in the 
Rytov theory as ${ }^{3}$

$$
D(r)=8 \pi^{2} k^{2} \int_{0}^{L} \int_{0}^{\infty} \kappa \Phi_{n}(\kappa, z) e^{-\Lambda L \kappa^{2}(1-z / L)^{2}} \cdot\left[I_{0}(\Lambda(1-z / L) \kappa r)-J_{0}([1-\bar{\Theta}(1-z / L) \kappa r)] d \kappa d z\right.
$$

where $J_{0}\left(I_{0}\right)$ is the (modified) Bessel function, and $\bar{\Theta}, \Lambda$ are the real and imaginary parts of the complex parameter $z /\left(z-q_{0}\right)$, respectively. For the results presented below this integral was evaluated numerically using the modified von Kármán refractive index PSD for each of the propagation scenarios considered.

Since a vertical propagation path through the Earth's atmosphere is highly asymmetric - with most of the turbulence located within the first few kilometers above ground level — we examine both the uplink and downlink paths in order to obtain a better understanding of how the different discretization schemes perform under these two important scenarios.

\subsection{Uplink atmospheric channel}

First we consider a $24 \mathrm{~km}$ vertical propagation path with the plane of the transmitter located at ground level. In this case, we assume a collimated Gaussian beam of diameter $6 \mathrm{~cm}$ and wavelength $\lambda=1.55 \mu \mathrm{m}$ representing a beam at the exit aperture of a ground-based transmitter. We use an $N \times N$ sampling grid with $N=1024$ and $\Delta x=\Delta y=1.9654 \mathrm{~mm}$. For the results presented in this section 200 simulations were performed to obtain the desired statistical convergence.

\subsubsection{Uplink wave structure function}

In Figures 4-5 the behavior of the wave structure function is shown at all spatial separations on a logarithmic scale against the behavior predicted from the Rytov theory (15) for the continuous turbulence profile. The solution to (15) for the path-averaged constant profile $\bar{C}_{n}^{2}(z)=\bar{C}_{n}^{2}=(1 / L) \int_{0}^{L} C_{n}^{2}\left(z^{\prime}\right) d z^{\prime}$ is also given for reference.

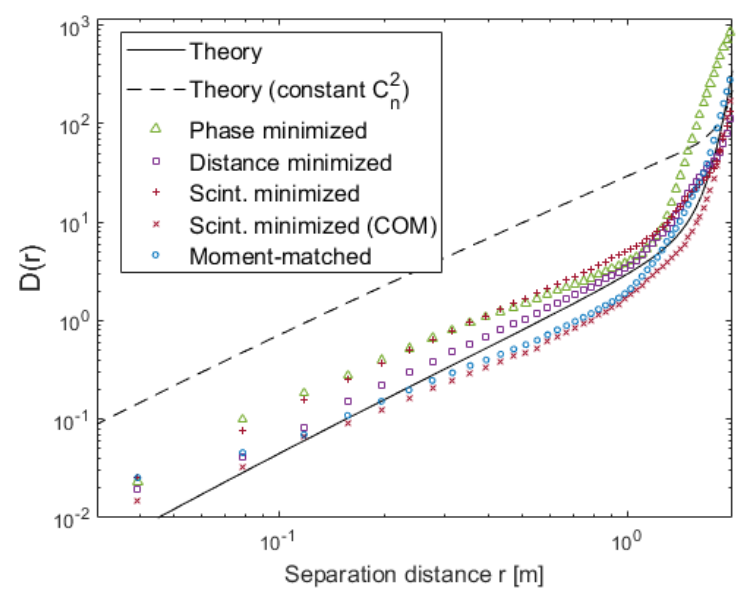

Figure 4. Wave structure function for uplink HV-21 profile with ground turbulence $C_{0}=1.7 \cdot 10^{-14} \mathrm{~m}^{-2 / 3}$.

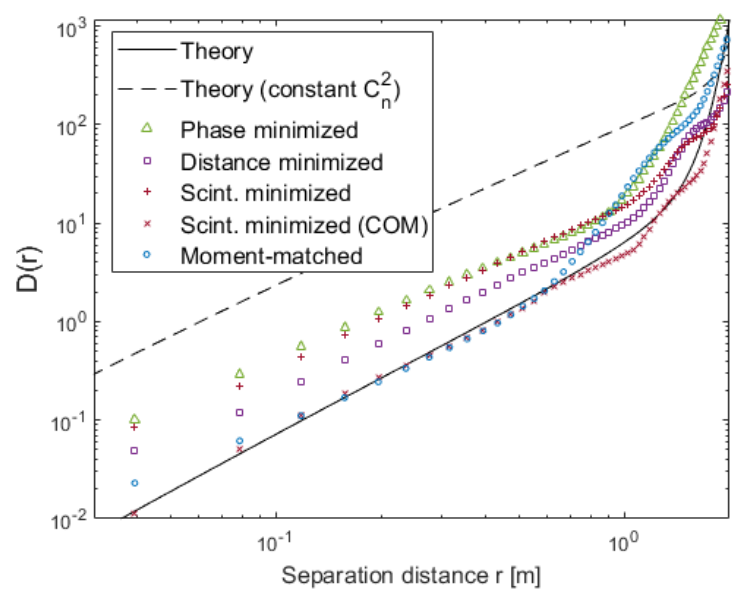

Figure 5. Wave structure function for uplink HV-21 profile with ground turbulence $C_{0}=6.8 \cdot 10^{-14} \mathrm{~m}^{-2 / 3}$.

At the higher turbulence level, the center of mass scintillation-minimized discretization yielded the closest agreement with the predicted wave structure function. At small spatial scales the moment-matched segmentation gave almost identical results, but exhibited an anomalous transition at a separation of $60 \mathrm{~cm}$, anticipating by $40 \mathrm{~cm}$ the expected transition at the beam edge separation of $1 \mathrm{~m}$. A similar but much smaller effect of this nature was also visible in the structure functions obtained from the other discretizations. In weaker turbulence $\left(C_{0}=1.7 \cdot 10^{-14} \mathrm{~m}^{-2 / 3}\right)$ the center of mass and moment-matched discretizations yielded the smallest absolute error at the smaller scales up to about $30 \mathrm{~cm}$; however, the centered distance-minimized discretization yielded a smaller error at the larger scales. 


\subsubsection{Uplink mean irradiance}

In contrast to the downlink path studied below, severe turbulence near the source leads to significant beam spreading on the uplink. For a unit-amplitude Gaussian beam with beam radius $w_{0}$ at the input plane the mean irradiance profile at the receiver plane can be approximated by a Gaussian ${ }^{3}$

$$
\langle I(r)\rangle \simeq \frac{w_{0}^{2}}{w_{e}^{2}} \exp \left(-\frac{2 \rho^{2}}{w_{e}^{2}}\right)
$$

where $w_{e}$ is the effective long-term spot radius given in the Rytov approximation as $w_{e}=w \sqrt{1+T}$ with $w=w(L)$ the diffraction-limited beam radius at the output plane and

$$
T=4 \pi^{2} k^{2} \int_{0}^{L} \int_{0}^{\infty} \kappa \Phi_{n}(\kappa, z)\left(1-e^{-2 \kappa^{2}(L-z)^{2} / k^{2} w^{2}}\right) d \kappa d z
$$

This is evaluated numerically and plotted in Figures 6-7 against the mean irradiance obtained from each discretization normalized by the on-axis irradiance after propagation in vacuum.

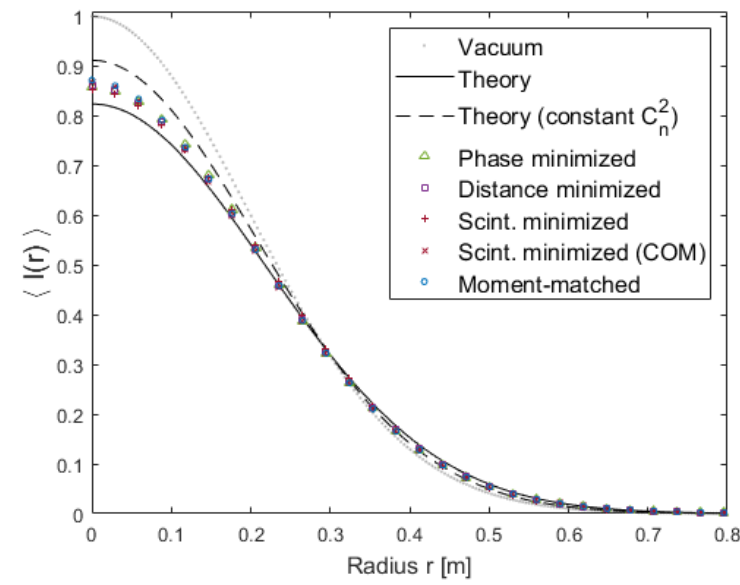

Figure 6. Mean irradiance for uplink HV-21 profile with Figure 7. Mean irradiance for uplink HV-21 profile with ground turbulence $C_{0}=1.7 \cdot 10^{-14} \mathrm{~m}^{-2 / 3}$.

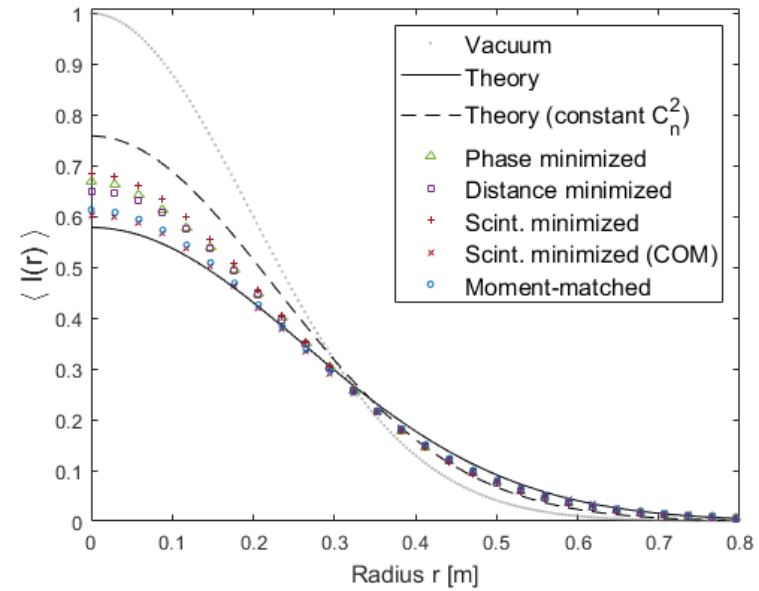

ground turbulence $C_{0}=6.8 \cdot 10^{-14} \mathrm{~m}^{-2 / 3}$.

The mean irradiance expected by the Rytov theory with path-averaged $C_{n}^{2}$ profile is shown for comparison. All of the discretizations yield a similar mean irradiance profile in the weaker turbulence profile; however, the center-of-mass scintillation-minimized and moment-matched discretizations show the closest agreement at the higher turbulence level while the other discretizations trended toward the mean irradiance expected from the path-averaged turbulence profile.

\subsubsection{Uplink scintillation index}

The scintillation index of an optical field with intensity $I(\boldsymbol{\rho})=|u(\boldsymbol{\rho}, L)|^{2}$ is defined by

$$
\sigma_{I}^{2}(\boldsymbol{\rho})=\frac{\left\langle I^{2}(\boldsymbol{\rho})\right\rangle-\langle I(\boldsymbol{\rho})\rangle^{2}}{\langle I(\boldsymbol{\rho})\rangle^{2}}=\exp \left(4\left\langle\chi^{2}\right\rangle\right)-1 .
$$

Unfortunately, the Rytov approximation for the scintillation index given by the integral (14) is not valid on the uplink propagation path considered here since the irradiance fluctuations near the beam edge are large even for relatively small ground turbulence levels $C_{0}$. Nevertheless, we show in Figure 8 the on-axis scintillation index obtained from the different discretizations at a range of ground turbulence levels.

We note that the moment-matched and center of mass scintillation-minimized discretizations yield similar results for the on-axis scintillation index, as do the distance- and phase-minimized discretizations, though the latter resulted in significantly more scintillation, while the centered scintillation-minimized screens resulted in even larger on-axis scintillation at the receiver plane. 


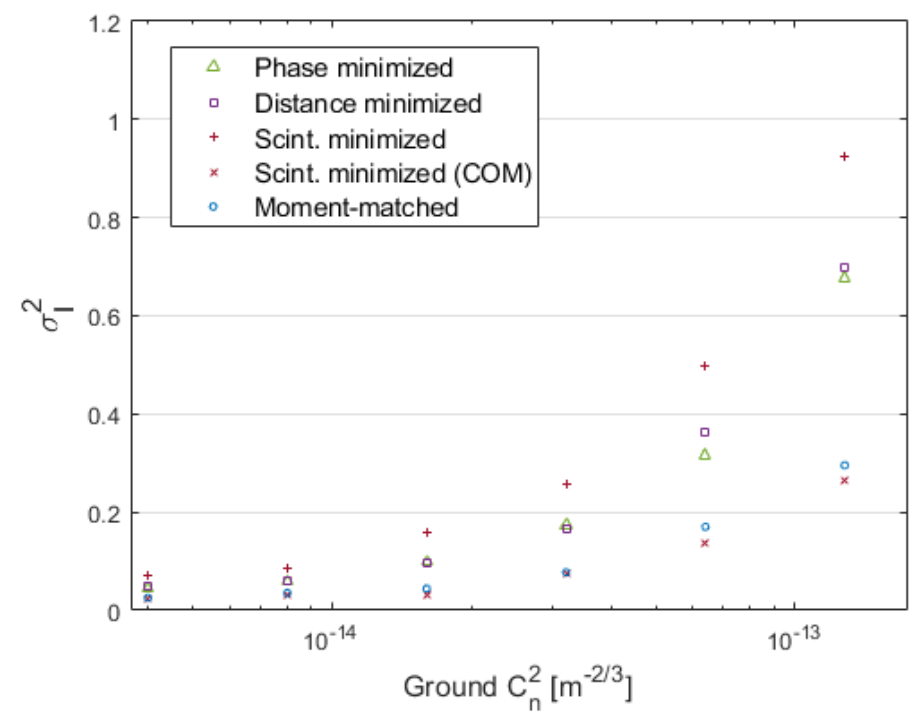

Figure 8. On-axis scintillation index for the uplink HV-21 turbulence profile as a function of the ground level turbulence parameter.

\subsection{Downlink atmospheric channel}

For the downlink channel the optical field at the source plane is taken to be a collimated Gaussian beam of diameter $2 \mathrm{~m}$ at a wavelength $\lambda=1.55 \mu \mathrm{m}$. Due to the large transverse size of the beam the sampling grid is taken with $N=4096$ points along each side and a separation distance $\Delta x=\Delta y=0.9827 \mathrm{~mm}$.

\subsubsection{Downlink wave structure function}

The results of 200 simulations were analyzed and the wave structure function computed within a $1 \mathrm{~m}$ window centered on the optical axis of the beam. For the downlink, the wave structure function computed via the first order Rytov theory (15) depends to an excellent approximation only on the total integrated turbulence

$$
D(r) \simeq 6.88\left(\frac{r}{r_{0}}\right)^{5 / 3}
$$

with $r_{0}=\left(0.423 k^{2} \int_{0}^{L} C_{n}^{2}(z) d z\right)^{-3 / 5}$. In Figures 9-10 we plot the wave structure function for each discretization on a logarithmic scale against the theoretical result (15).

At both turbulence levels the centered scintillation-minimized phase screens show slightly better agreement with the theoretical expression (15) though the differences in the schemes becomes more difficult to distinguish in the more turbulent conditions.

A more highly resolved look at the smaller scale behavior is also given in Figures 11-12 in the form of the modulus of the complex degree of coherence $\mu(r)=\exp \left(-\frac{1}{2} D(r)\right)$ where the spatial coherence radius $\rho_{0}=r_{0} / 2.1$ can be read off as the $1 / e$ point $\mu\left(\rho_{0}\right)=1 / e$. In weaker turbulence all of the approaches yield the theoretical value of $\rho_{0}=9.2 \mathrm{~cm}$ within an absolute error of $2.6 \mathrm{~cm}$, though the centered scintillation-minimized and momentedmatched discretizations yield the closest agreement with an error of 1.5 and $1.4 \mathrm{~cm}$, respectively. At stronger turbulence the simulations yield the spatial coherence radius $\rho_{0}=4.5 \mathrm{~cm}$ within an absolute error of $1 \mathrm{~cm}$ with the centered scintillation- and distance-minimized screens yielding an error within $0.3 \mathrm{~cm}$.

\subsubsection{Downlink scintillation index}

For a Gaussian beam in the regime of weak irradiance fluctuations $\left(\sigma_{I}^{2} \lesssim 0.25\right)$ the Rytov approximation gives the on-axis scintillation index via the integral (14); however, we note that for a Gaussian beam on the downlink 


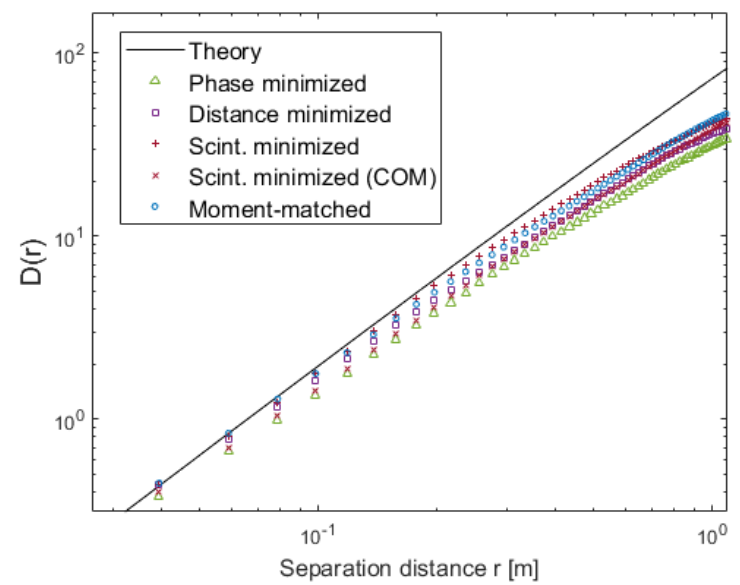

Figure 9. Wave structure function for downlink HV-21 profile with ground turbulence $C_{0}=1.7 \cdot 10^{-14} \mathrm{~m}^{-2 / 3}$.

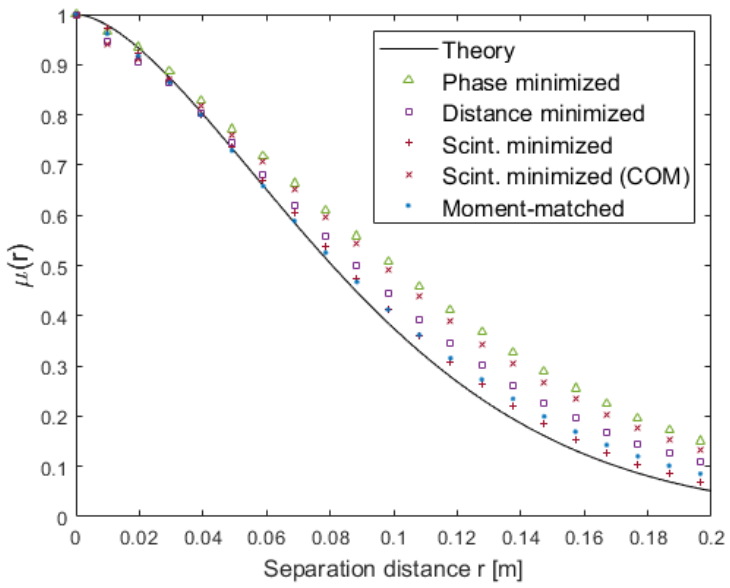

Figure 11. Modulus of the complex degree of coherence for downlink HV-21 profile with ground turbulence $C_{0}=1.7$. $10^{-14} \mathrm{~m}^{-2 / 3}$.

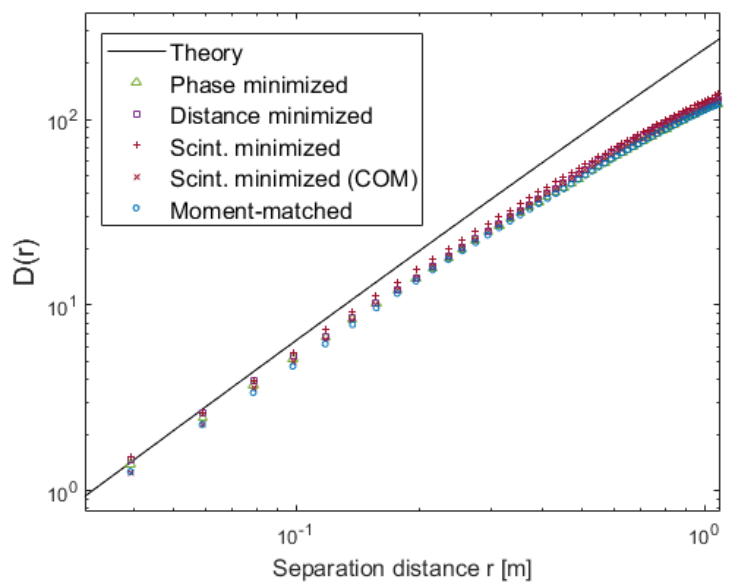

Figure 10. Wave structure function for downlink HV-21 profile with ground turbulence $C_{0}=6.8 \cdot 10^{-14} \mathrm{~m}^{-2 / 3}$.

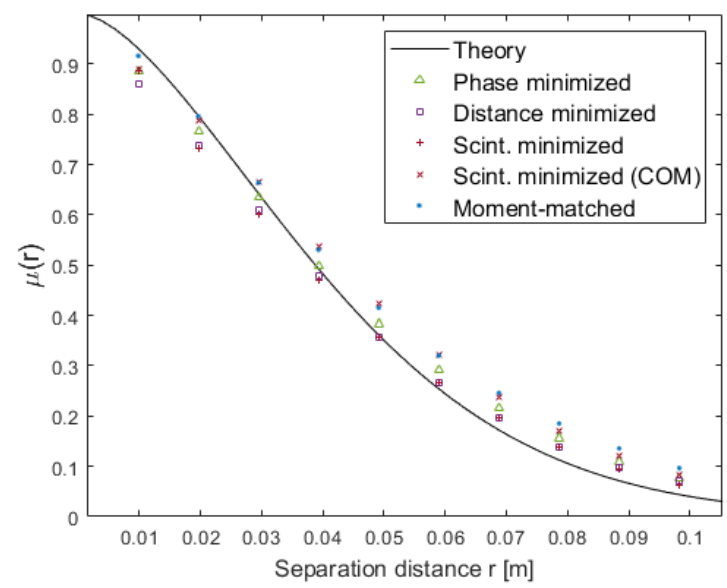

Figure 12. Modulus of the complex degree of coherence for downlink HV-21 profile with ground turbulence $C_{0}=6.8$. $10^{-14} \mathrm{~m}^{-2 / 3}$.

path the scintillation index is roughly constant up to the beam edge and is well approximated by the plane wave expression (i.e. the Rytov variance)

$$
\sigma_{R}^{2}=2.25 k^{7 / 6} \int_{0}^{L} C_{n}^{2}(z)(L-z)^{5 / 6} d z .
$$

The resulting scintillation index for the Hufnagel-Valley turbulence profile with rms wind $v=21 \mathrm{~m} / \mathrm{s}$ at a range of values for the ground level turbulence parameter ranging from the weak fluctuation regime to the onset of strong fluctuations is shown in Figure 13.

As a point of reference, we also show the scintillation index for a constant $C_{n}^{2}$ profile with the equivalent path-averaged $C_{n}^{2}$ compared to the corresponding Hufnagel-Valley profile. However, for the path-averaged profile the onset of strong fluctuations occurs earlier so we use a model for the on-axis scintillation index introduced by Andrews et al. ${ }^{12}$

$$
\sigma_{I}^{2}=\exp \left(\frac{0.49 \sigma_{R}^{2}}{\left(1+1.11 \sigma_{R}^{12 / 5}\right)^{7 / 6}}+\frac{0.51 \sigma_{R}^{2}}{\left(1+0.69 \sigma_{R}^{12 / 5}\right)^{5 / 6}}\right)-1
$$

which accounts for saturation in the strong fluctuation regime. By averaging the scintillation index over the central portion of the sampling grid of $1 \mathrm{~m}$ diameter we obtain sufficient statistical convergence using 20 simulations 
at each turbulence level.

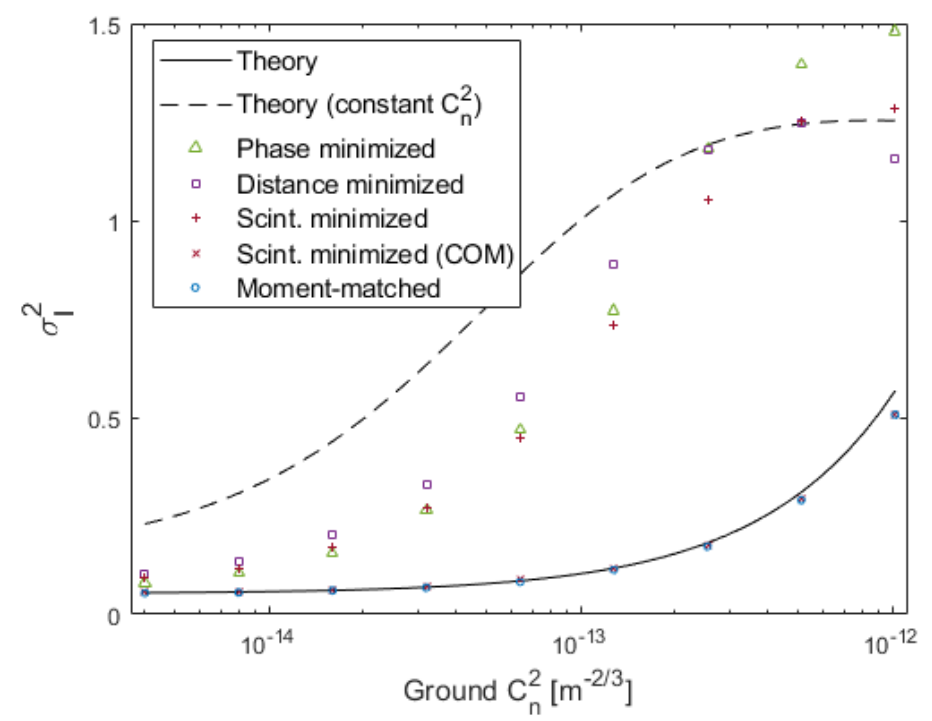

Figure 13. Scintillation index for the downlink HV-21 turbulence profile as a function of the ground level turbulence parameter. (Note: the scint.minimized $(\mathrm{COM})$ and moment-matched results are almost identical)

The center of mass scintillation-minimized and moment-matched models yielded excellent agreement with the theoretically-predicted scintillation for the continuous turbulence profile. As the ground-level turbulence is increased, the other discretizations all over-estimate the scintillation index by values approaching (and even exceeding) the predicted scintillation for the corresponding constant turbulence profile. Notably, the centered distance- and centered scintillation-minimized discretizations appear to exhibit saturation at roughly the turbulence level where saturation is expected from the path-averaged constant turbulence profiles.

\section{SUMMARY}

For the atmospheric channels described above, we examined several discretization algorithms for the segmentation of the propagation path and placement of the phase screens. For those statistics which depend (in the Rytov theory) on the higher moments of the continuous turbulence profile we found that an algorithm which split the propagation path based on minimizing the contribution of each segment to the total scintillation and located the phase screens at the turbulent center of mass in each segment (as defined in Section 3) exhibits the closest agreement with the statistics expected from the Rytov theory. This discretization yielded comparable results to a four-layer model introduced by Troxel et al. for this particular propagation path known to yield close agreement to the optical transfer function of the continuous turbulence profile. Other discretizations of the propagation path which located the screens at the center of each segment produced results tending toward those expected from the path-averaged turbulence profile, and thus tended to yield better agreement with the Rytov theory for those statistics which depend only on the zeroth moment of the continuous turbulence profile.

\section{ACKNOWLEDGEMENTS}

This work is supported by the NASA Space Communications and Navigation (SCaN) Program and the Glenn Research Center Communications \& Intelligent Systems Division. This work was also supported by the Convergent Aeronautics Solutions (CAS) project. 


\section{APPENDIX A.}

\section{A.1 Phase power spectral density for path-dependent refractive index statistics}

In this appendix, we deduce the two-dimensional phase power spectral density (10) assuming a refractive index power spectral density of the form $\Phi_{n}(\boldsymbol{\kappa}, z)=\sigma_{n}^{2}(z) \hat{\Phi}_{n}(\boldsymbol{\kappa})$. The starting point is the expression (9), repeated here for convenience,

$$
\Phi_{\phi}\left(\boldsymbol{\kappa}_{\rho}\right)=k^{2} \int_{z_{i}}^{z_{i+1}} \int_{z_{i}}^{z_{i+1}} \int_{-\infty}^{\infty} e^{i \kappa_{z}\left(\zeta_{2}-\zeta_{1}\right)} \Phi_{n}\left(\boldsymbol{\kappa}_{\rho}, \kappa_{z}, \frac{\zeta_{1}+\zeta_{2}}{2}\right) d \kappa_{z} d \zeta_{1} d \zeta_{2}
$$

where $\boldsymbol{\kappa}=\left(\boldsymbol{\kappa}_{\rho}, \kappa_{z}\right)$ denotes the splitting into transverse and longitudinal components. To evaluate this, we first introduce the change of variables $\zeta_{ \pm}=\left(\zeta_{2} \pm \zeta_{1}\right) / 2$ and set $\zeta_{0}=\left(z_{i}+z_{i+1}\right) / 2$ whereupon the integral above becomes

$$
\begin{aligned}
\Phi_{\phi}\left(\boldsymbol{\kappa}_{\rho}\right) & =2 k^{2} \int_{-\infty}^{\infty}\left(\int_{z_{i}}^{\zeta_{0}} \int_{z_{i}-\zeta_{+}}^{\zeta_{+}-z_{i}} e^{2 i \kappa_{z} \zeta_{-}} \Phi_{n}\left(\boldsymbol{\kappa}, \zeta_{+}\right) d \zeta_{-} d \zeta_{+}+\int_{\zeta_{0}}^{z_{i+1}} \int_{\zeta_{+}-z_{i+1}}^{z_{i+1}-\zeta_{+}} e^{2 i \kappa_{z} \zeta_{-}} \Phi_{n}\left(\boldsymbol{\kappa}, \zeta_{+}\right) d \zeta_{-} d \zeta_{+}\right) d \kappa_{z} \\
& =2 k^{2} \int_{-\infty}^{\infty}\left(\int_{z_{i}}^{\zeta_{0}} \Phi_{n}\left(\boldsymbol{\kappa}, \zeta_{+}\right) \frac{\sin \left(2 \kappa_{z}\left(\zeta_{+}-z_{i}\right)\right)}{\kappa_{z}} d \zeta_{+}+\int_{\zeta_{0}}^{z_{i+1}} \Phi_{n}\left(\boldsymbol{\kappa}, \zeta_{+}\right) \frac{\sin \left(2 \kappa_{z}\left(z_{i+1}-\zeta_{+}\right)\right)}{\kappa_{z}} d \zeta_{+}\right) d \kappa_{z} .
\end{aligned}
$$

Introducing another change of variables $s=2\left(\zeta_{+}-z_{i}\right)$ in the first integral and $s=2\left(z_{i+1}-\zeta_{+}\right)$in the second we obtain

$$
\Phi_{\phi}\left(\boldsymbol{\kappa}_{\rho}\right)=k^{2} \int_{-\infty}^{\infty} \int_{0}^{\Delta z}\left[\Phi_{n}\left(\boldsymbol{\kappa}, z_{i}+\frac{s}{2}\right)+\Phi_{n}\left(\boldsymbol{\kappa}, z_{i+1}-\frac{s}{2}\right)\right] \frac{\sin \left(\kappa_{z} s\right)}{\kappa_{z}} d s d \kappa_{z} .
$$

Separating out the path-dependence $\Phi_{n}(\boldsymbol{\kappa}, z)=\sigma_{n}^{2}(z) \hat{\Phi}_{n}(\boldsymbol{\kappa})$ gives

$$
\Phi_{\phi}\left(\boldsymbol{\kappa}_{\rho}\right)=k^{2} \int_{0}^{\Delta z}\left[\sigma_{n}^{2}\left(z_{i}+\frac{s}{2}\right)+\sigma_{n}^{2}\left(z_{i+1}-\frac{s}{2}\right)\right]\left(\int_{-\infty}^{\infty} \hat{\Phi}_{n}\left(\boldsymbol{\kappa}_{\rho}, \kappa_{z}\right) \frac{\sin \left(\kappa_{z} s\right)}{\kappa_{z}} d \kappa_{z}\right) d s
$$

We have thus obtained the exact solution for the phase screen PSD in terms of the refractive index PSD, which we may rewrite in the form

$$
\Phi_{\phi}\left(\boldsymbol{\kappa}_{\rho}\right)=k^{2} \int_{0}^{\Delta z}\left[\sigma_{n}^{2}\left(z_{i}+\frac{s}{2}\right)+\sigma_{n}^{2}\left(z_{i+1}-\frac{s}{2}\right)\right] K_{n}\left(\boldsymbol{\kappa}_{\rho}, s\right) d s
$$

where the spatial filter function $K_{n}\left(\boldsymbol{\kappa}_{\rho}, s\right)$ depends on the distance $s / 2$ to the boundary of the segment $\left[z_{i}, z_{i+1}\right]$.

Note that if $L_{0}$ is the outer correlation length of the refractive index fluctuations then for $\kappa_{z} \ll 1 / L_{0}$ we have $\hat{\Phi}_{n}\left(\boldsymbol{\kappa}_{\rho}, \kappa_{z}\right) \simeq \hat{\Phi}_{n}\left(\boldsymbol{\kappa}_{\rho}, \kappa_{z}=0\right)$. Thus, for $s \gg L_{0}$ the kernel defining the spatial filter $K_{n}$ limits to a delta distribution $\sin \left(\kappa_{z} s\right) / \kappa_{z} \sim \pi \delta\left(\kappa_{z}\right)$ and we obtain $K_{n}\left(\boldsymbol{\kappa}_{\rho}, s\right) \simeq \pi \hat{\Phi}_{n}\left(\boldsymbol{\kappa}_{\rho}, \kappa_{z}=0\right)$. On the other hand, for $\kappa_{z} \gg 1 / l_{0}$ where $l_{0}$ is the inner correlation length of the fluctuations, one has $\Phi_{n}\left(\boldsymbol{\kappa}_{\rho}, \kappa_{z}\right) \simeq 0$ and hence for $s \ll l_{0}$ the filter function vanishes $K_{n}\left(\boldsymbol{\kappa}_{\rho}, s\right) \simeq 0$. Thus, (assuming $\Delta z>L_{0}$ ) the expression above can be approximated by

$$
\Phi_{\phi}\left(\boldsymbol{\kappa}_{\rho}\right) \simeq 2 \pi k^{2} \hat{\Phi}_{n}\left(\boldsymbol{\kappa}_{\rho}, 0\right) \int_{L_{0}}^{\Delta z} \sigma_{n}^{2}(z) d z+k^{2} \int_{l_{0}}^{L_{0}}\left[\sigma_{n}^{2}\left(z_{i}+\frac{s}{2}\right)+\sigma_{n}^{2}\left(z_{i+1}-\frac{s}{2}\right)\right] K_{n}\left(\boldsymbol{\kappa}_{\rho}, s\right) d s
$$

where $l_{0}$ and $L_{0}$ are rough order-of-magnitude estimates for the inner and outer correlation lengths which in practice may be chosen to ensure the validity of the approximations described above. Now, assuming that (1) the propagation distance is much larger than the outer correlation length $\Delta z \gg L_{0}$ and (2) the variance $\sigma_{n}^{2}(z)$ is not localized primarily within a distance on the order of $L_{0}$ from the segment boundary, the second integral representing contributions from refractive index fluctuations near the boundary is a small perturbation of the bulk integral given by the first term. Under these assumptions, we are thus led to the approximation (10) given in Section 2.

As a final remark, we note that some of the discretizations studied in Section 3 do not appear to satisfy these conditions, as the path segments near ground level are not much longer than the assumed correlation length 
$L_{0}=100 \mathrm{~m}$ and furthermore the refractive index fluctuations of the Hufnagel-Valley profile are highly localized within the first few hundred meters of ground level. In this case, the expression (10) is valid only up to the limits of the Markov approximation which assumes that the refractive index fluctuations are $\delta$-correlated in the direction of propagation and yields an alternative derivation of (10). ${ }^{6}$ Although this is a somewhat unphysical assumption, in certain cases analysis of the stochastic parabolic wave equation (1) based on the Markov approximation is known to yield reliable results for a number of statistical moments of the solutions and is the basis of the strong fluctuation theory. ${ }^{4}$

\section{A.2 Operator-splitting for path-dependent random background potential}

In this section, we study analytically the error introduced by the discretization in (4). The analysis in this section applies generally to any application of the operator splitting described by equations (3) and (4) to solve the Schrödinger equation (1) with a random background potential $n_{1}$. Our analysis is similar in spirit to that carried out by Spivack and Uscinski, ${ }^{1}$ but with particular attention to the path-dependent correlation statistics of $n_{1}$. Our approach is based on the Baker-Campbell-Hausdorff formula

$$
e^{X} e^{Y}=\exp \left(X+Y+\frac{1}{2}[X, Y]+\frac{1}{12}([X,[X, Y]]+[Y,[Y, X]])+\ldots\right)
$$

where the remaining terms involve higher-order commutators of $X$ and $Y$. More generally, this implies

$$
e^{\alpha X} e^{Y} e^{(1-\alpha) X}=\exp \left(X+Y+\left(\alpha-\frac{1}{2}\right)[X, Y]+\frac{1}{12}[Y,[Y, X]]+\frac{1}{12}\left(5 \alpha^{2}+5 \alpha+1\right)[X,[X, Y]]+\ldots\right)
$$

Applying this to (4) we obtain

$$
e^{i \alpha(\Delta z / k) \hat{A}} e^{i \phi} e^{i(1-\alpha)(\Delta z / k) \hat{A}}=\exp \left(i \int_{z_{i}}^{z_{i+1}} H(z) d z+\left(\frac{1}{2}-\alpha\right)(\Delta z / k)\left(k \Sigma_{n}\right)[\hat{A}, \hat{\phi}]+\ldots\right) .
$$

where $\hat{A}=k A$ is the normalized free-space propagator and $\hat{\phi}$ is the normalized phase defined in Section 2. Note that the first order error term can be eliminated by setting $\alpha=1 / 2$.

At this point, it is important to note that the higher order commutators of $\hat{A}$ and $\hat{\phi}$ may not remain wellcontrolled. Indeed, if we assume the solution $u(\boldsymbol{\rho}, z)$ vanishes outside some domain of finite transverse radius $|\boldsymbol{\rho}|=R$ and expand $\hat{\phi}$ in a Fourier series $\hat{\phi}(\boldsymbol{\rho})=\sum_{n, m} c_{n, m} e^{i \boldsymbol{\kappa}_{n, m} \cdot \boldsymbol{\rho}}$ with $\boldsymbol{\kappa}_{n, m}=(n \pi / R, m \pi / R)$ we observe that the Fourier coefficients of $\hat{A} \hat{\phi}$ given by

$$
\hat{A} \hat{\phi}=-\frac{1}{2} \sum_{n, m} \boldsymbol{\kappa}_{n, m}^{2} c_{n, m} e^{i \boldsymbol{\kappa}_{n, m} \cdot \boldsymbol{\rho}}
$$

include a scaling by a factor of $\boldsymbol{\kappa}_{n, m}^{2} / 2$. Noting that $\left\langle\left|c_{n, m}\right|^{2}\right\rangle=(\pi / R) \hat{\Phi}_{n}\left(\boldsymbol{\kappa}_{n, m}, 0\right)$ we are motivated to introduce the dimensionless operator $\hat{A}_{0}=\hat{A} l_{0}^{2}$ where $l_{0}$ is the inner correlation length of the refractive index fluctuations (i.e. $\left.\hat{\Phi}_{n}\left(2 \pi / l_{0}, 0\right) \ll 1\right)$. One thus retains some control over the higher order commutators of $\hat{A}_{0}$ and $\hat{\phi}$ and so the second order error can be written in the form

$$
\epsilon=-\frac{i}{12}\left(\left(\Delta z / k l_{0}^{2}\right)\left(k \Sigma_{n}\right)^{2}\left[\hat{\phi},\left[\hat{\phi}, \hat{A}_{0}\right]\right]+\left(5 \alpha^{2}+5 \alpha+1\right)\left(\Delta z / k l_{0}^{2}\right)^{2}\left(k \Sigma_{n}\right)\left[\hat{A}_{0},\left[\hat{A}_{0}, \hat{\phi}\right]\right]\right) .
$$

More generally, with this normalization the $n$-th order error in the exponent of the propagator carries factors of the form $\left(\Delta z / k l_{0}^{2}\right)^{p}\left(k \Sigma_{n}\right)^{q}$ where $p+q=n+1$. However, it should be noted that the assumption that the total error in the propagator introduced by (19) is small depends also on the solution space on which the commutators of $A$ and $\phi$ act, a point noted by Spivack. ${ }^{13}$ Indeed, the very convergence of the exponent in (19) depends crucially on the solution space on which the operator acts, an issue we have left aside in this analysis.

Nonetheless, in order to minimize the error introduced by the second part of the phase screen approximation (4) the observations above motivate one to consider the discretizations $0<z_{1}<\ldots<z_{n}=L$ which minimize some combination of the propagation distance $\Delta z / k l_{0}^{2}$ and integrated variance $k \Sigma_{n}$. 


\section{REFERENCES}

1. M. Spivack and B. Uscinski, "The split-step solution in random wave propagation," Journal of Computational and Applied Mathematics 27(3), pp. 349 - 361, 1989.

2. R. L. Fante, "Electromagnetic beam propagation in turbulent media.," IEEE Proceedings 63, pp. 1669-1692, Dec 1975.

3. L. C. Andrews and R. L. Phillips, Laser Beam Propagation through Random Media, SPIE Publications, Bellingham, Washington, 2 ed., 2005.

4. R. M. Manning, "The atmospheric mutual coherence function from the first and second Rytov approximations and its comparison to that of strong fluctuation theory," Tech. Rep. NASA/TM-2011-216974, NASA Langley Research Center, Hampton, Virginia, January 2011.

5. W. A. Coles, J. P. Filice, R. G. Frehlich, and M. Yadlowsky, "Simulation of wave propagation in threedimensional random media," Appl. Opt. 34, pp. 2089-2101, Apr 1995.

6. J. M. Martin and S. M. Flatté, "Intensity images and statistics from numerical simulation of wave propagation in 3-d random media," Appl. Opt. 27, pp. 2111-2126, Jun 1988.

7. J. D. Schmidt, Numerical Simulation of Optical Wave Propagation with Examples in MATLAB, SPIE, Bellingham, Washington, 2010.

8. S. E. Troxel, B. M. Welsh, and M. C. Roggemann, "Off-axis optical transfer function calculations in an adaptive-optics system by means of a diffraction calculation for weak index fluctuations.," Journal of the Optical Society of America A 11, pp. 2100-2111, Jul 1994.

9. A. Ishimaru, "Fluctuations of a focused beam wave for atmospheric turbulence probing," Proceedings of the IEEE 57(4), pp. 407-414, 1969.

10. H. Hemmati, Deep Space Optical Communications, John Wiley and Sons, Hoboken, New Jersey, 2006.

11. R. Frehlich, "Simulation of laser propagation in a turbulent atmosphere," Appl. Opt. 39, pp. 393-397, Jan 2000 .

12. L. C. Andrews, R. L. Phillips, and C. Y. Young, "Scintillation model for a satellite communication link at large zenith angles," Optical Engineering 39(12), pp. 3272 - 3280, 2000.

13. M. Spivack, "Operator splitting for the random wave moment equations," Applied Mathematics Letters 3(2), pp. $87-91,1990$.

14. H. G. Booker, J. A. Ferguson, and H. O. Vats, "Comparison between the extended-medium and the phasescreen scintillation theories," Journal of Atmospheric and Terrestrial Physics 47(4), pp. 381 - 399, 1985.

15. L. C. Andrews, R. L. Phillips, and A. R. Weeks, "Propagation of a Gaussian-beam wave through a random phase screen," Waves in Random Media 7, pp. 229-244, Apr 1997. 